\section{Successful Endoscopic Retrieval of an Impacted Trapezoid Basket with Severed Traction Wires}

A wire-guided trapezoid lithotriptor has been introduced recently for the endoscopic extraction of bile duct stones. We describe a case of an impacted trapezoid basket with severed traction wires, and its successful removal from a patient with choledocholithiasis.

A 83-year-old man, who had previously undergone a cholecystectomy, was suspected on ultrasonography to have a bile duct stone. Endoscopic retrograde cholangiopancreatography was performed using a TJF-240 Olympus video duodenoscope. Cholangiography showed a stone in the common bile duct. Following sphincterotomy, a trapezoid lithotriptor (M00510880, basket length $5 \mathrm{~cm}$, width $2.5 \mathrm{~cm}$; Boston Scientific/Microvasive, Watertown, Massachusetts, USA) was used to extract the stone, which then became impacted in the basket. In the attempt to crush the impacted stone, the traction wires within the sheath snapped and became detached distally, so that they were exposed in the mid-esophagus (Figure 1). Rat-tooth forceps were used to push the wires into the stomach. The wires were then grasped, twisted at the ends for a firmer grip by rotating the scope, pulled into the working channel, and locked in by the elevator (Figure $\mathbf{2}$ ). The wires and the trapezoid basket containing the stone were successfully retrieved through the mouth by withdrawing the scope.

We have increasingly been using the trapezoid lithotriptor in our unit because of its ability to crush stones which cannot be extracted through the sphincterotomy. We believe the traction wires ruptured during lithotripsy in this patient because the stone was too hard to be crushed. The case was complicated because the severed wires splayed outwards within the esophageal lumen, making it difficult to extract them without traumatizing the esophagus.

The entrapment of a broken basket in the bile duct is an unusual complication of

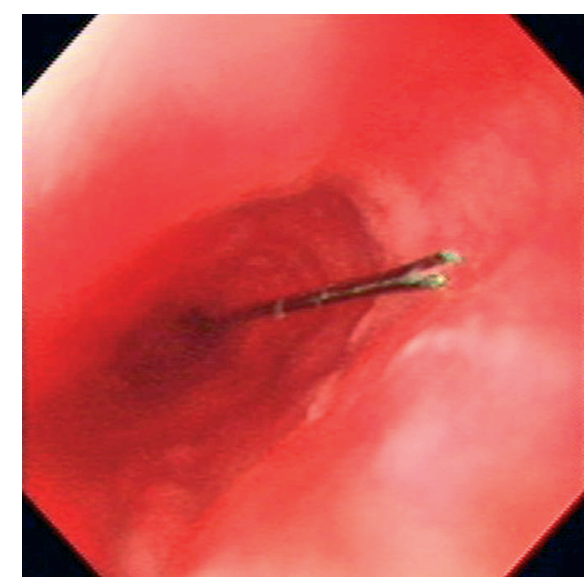

Figure 1 The ruptured traction wires, with their ends splayed, in the mid-esophageal lumen.

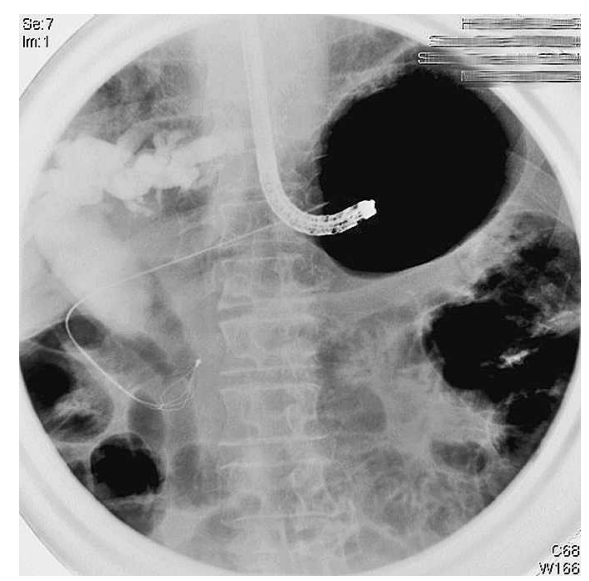

Figure 2 A rat-tooth forceps was used to grasp the severed traction wires before they were retrieved.

endoscopic stone removal [1-4]. As far as we are aware, successful endoscopic retrieval of ruptured traction wires, which occurred as a complication of endoscopic stone extraction, has not been previously described.

\section{C. B. Caluag, K. Y. Ho}

Endoscopic Centre, National University Hospital, Singapore.
References

${ }^{1}$ Schutz SM, Chinea C, Friedrichs P. Successful endoscopic removal of a severed, impacted Dormia basket. Am J Gastroenterol 1997; 92: 679-681

2 Utsunomiya K, Tamada K, Tomiyama Tet al. Calcified bile duct stone not crushable by endotriptor or mechanical lithotriptor: case report. Surg Laparosc Endosc Percutan Tech 2002; 12: 184- 186

${ }^{3}$ Sauter G, Sackmann M, Holl Jet al. Dormia baskets impacted in the bile duct: release by extracorporeal shock-wave lithotripsy. Endoscopy 1995; 27: $384-$ 387

${ }^{4}$ van Dullemen H, Stuifbergen WN, Juttmann JR, van der Werken C. Simple release of an impacted Dormia basket during endoscopic bile duct stone extraction. Endoscopy 1993; 25: 374

\section{Corresponding Author}

\section{K.-Y. Ho, M.D.}

Department of Medicine National University Hospital

5 Lower Kent Ridge Road 119074 Singapore

Singapore

Fax: $\quad+65-67794112$

E-mail: mdchoky@nus.edu.sg 